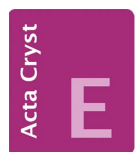

CRYSTALLOGRAPHIC COMMUNICATIONS

ISSN 2056-9890

Received 24 May 2016

Accepted 28 May 2016

Edited by J. Simpson, University of Otago, New Zealand

Keywords: crystal structure; indole; $\mathrm{N}-\mathrm{H}$... hydrogen bonds; inversion dimers; weak interactions.

CCDC references: 1482360; 1482359

Supporting information: this article has supporting information at journals.iucr.org/e

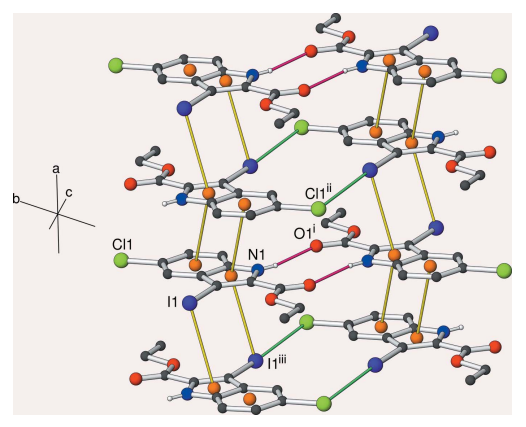

OPEN $\odot$ ACCESS

\section{Weak interactions in the crystal structures of two indole derivatives}

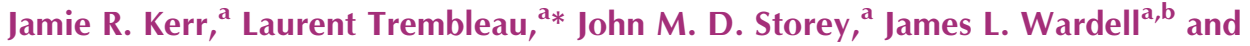 \\ William T. A. Harrison ${ }^{\text {a* }}$
}

${ }^{\mathbf{a} D}$ Department of Chemistry, University of Aberdeen, Meston Walk, Aberdeen AB24 3UE, Scotland, and ${ }^{\mathbf{b}}$ Fundação
Oswaldo Cruz, Instituto de Tecnologia em Fármacos-Far Manguinhos, 21041-250 Rio de Janeiro, RJ, Brazil.
${ }^{*}$ Correspondence e-mail: I.trembleau@abdn.ac.uk, w.harrison@abdn.ac.uk

We describe the syntheses and crystal structures of two indole derivatives, namely a second monoclinic polymorph of ethyl 5-chloro- $1 H$-indole-2-carboxylate $\mathrm{C}_{11} \mathrm{H}_{10} \mathrm{ClNO}_{2}$, (I), and ethyl 5-chloro-3-iodo- $1 \mathrm{H}$-indole-2-carboxylate, $\mathrm{C}_{11} \mathrm{H}_{9} \mathrm{ClINO}_{2}$, (II). In their crystal structures, both compounds form inversion dimers linked by pairs of $\mathrm{N}-\mathrm{H} \cdots \mathrm{O}$ hydrogen bonds, which generate $R_{2}^{2}(10)$ loops. The dimers are linked into double chains in (I) and sheets in (II) by a variety of weak interactions, including $\pi-\pi$ stacking, $\mathrm{C}-\mathrm{I} \cdots \pi, \mathrm{C}-\mathrm{Cl}-\pi$ interactions and $\mathrm{I} \cdots \mathrm{Cl}$ halogen bonds.

\section{Chemical context}

As part of our ongoing synthetic, biological (Kerr, 2013) and structural studies (Kerr et al., 2016) of variously substituted indole derivatives, we now report the syntheses and crystal structures of ethyl 5-chloro- $1 H$-indole-2-carboxylate (I) and ethyl 5-chloro-3-iodo- $1 H$-indole-2-carboxylate (II), which differ in the substituent ( $\mathrm{H}$ or I) at the 3-position of the ring system. Compound (I) is a second monoclinic polymorph of the recently described 5-chloro- $1 H$-indole-2-carboxylate $(\mathrm{Wu}$ et al., 2013).

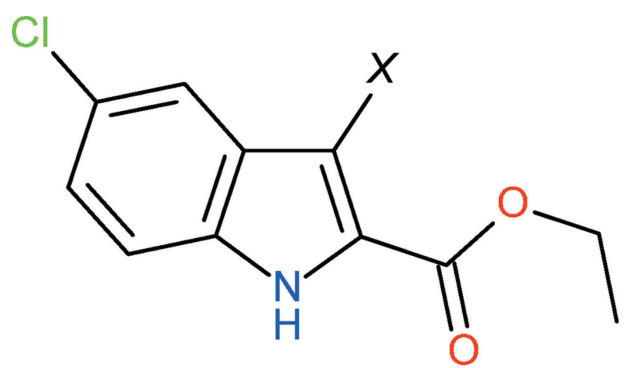
(I) $X=\mathrm{H}$
(II) $X=1$

\section{Structural commentary}

Compound (I) crystallizes in space group $P 2_{1} / n$ with one molecule in the asymmetric unit (Fig. 1). The dihedral angle between the mean plane of the $\mathrm{N} 1 / \mathrm{C} 1-\mathrm{C} 8$ indole ring system (r.m.s. deviation $=0.010 \AA$ ) and the $\mathrm{C} 9 / \mathrm{O} 1 / \mathrm{O} 2$ grouping is $2.4(2)^{\circ}$. The chlorine atom deviates from the indole plane by 0.0625 (14) $\AA$. The $\mathrm{C} 8-\mathrm{C} 9-\mathrm{O} 1-\mathrm{C} 10$ torsion angle of 


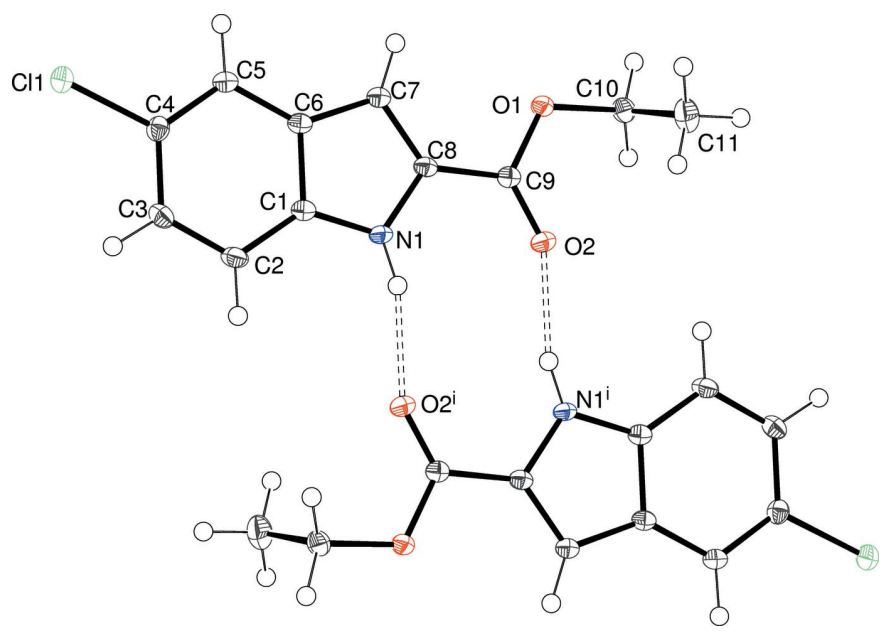

Figure 1

The molecular structure of (I) showing 50\% displacement ellipsoids. Also shown with double-dashed lines are the pair of intermolecular $\mathrm{N}-\mathrm{H} \cdots \mathrm{O}$ hydrogen bonds to a nearby molecule related by inversion symmetry, which generate an $R_{2}^{2}(10)$ loop. Symmetry code: (i) $1-x, 2-y, 1-z$.

$-178.86(11)^{\circ}$ indicates an anti conformation about the C9$\mathrm{O} 1$ bond, whereas the $\mathrm{C} 9-\mathrm{O} 1-\mathrm{C} 10-\mathrm{C} 11$ torsion angle is $-81.73(14)^{\circ}$ and $\mathrm{C} 11$ projects from the mean plane of the other non-hydrogen atoms by 1.298 (2) $\AA$.

In the structure reported by $\mathrm{Wu}$ et al. (2013), (CCDC refcode VIHMUW), the same molecule also crystallizes in space group $P 2_{1} / n[a=10.570(3), b=5.6165(15), c=$ 18.091 (5) $\left.\AA, \beta=105.681(4)^{\circ}, V=1034.0(5) \AA^{3}, Z=4\right]$ : the only significant conformational difference compared to (I) is (using our atom-labelling scheme) the $\mathrm{C} 9-\mathrm{O} 1-\mathrm{C} 10-\mathrm{C} 11$

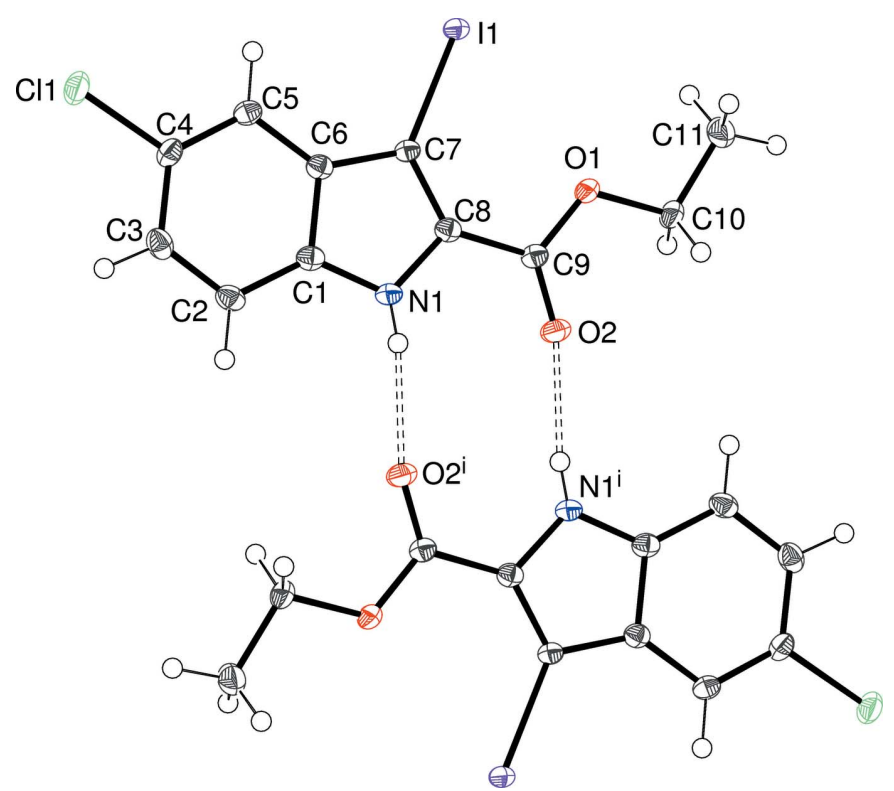

Figure 2

The molecular structure of (II) showing 50\% displacement ellipsoids. Also shown with double-dashed lines are the pair of intermolecular $\mathrm{N}-$ $\mathrm{H} \cdots \mathrm{O}$ hydrogen bonds to a nearby molecule related by inversion symmetry, which generate an $R_{2}^{2}(10)$ loop. Symmetry code: (i) $-x, 2-y$, $1-z$.
Table 1

Hydrogen-bond geometry $\left(\AA,^{\circ}\right)$ for (I).

\begin{tabular}{lllll}
\hline$D-\mathrm{H} \cdots A$ & $D-\mathrm{H}$ & $\mathrm{H} \cdots A$ & $D \cdots A$ & $D-\mathrm{H} \cdots A$ \\
\hline $\mathrm{N} 1-\mathrm{H} 1 \cdots \mathrm{O} 2^{\mathrm{i}}$ & $0.878(17)$ & $1.977(17)$ & $2.8288(15)$ & $163.0(15)$ \\
\hline
\end{tabular}

Symmetry code: (i) $-x+1,-y+2,-z+1$.

torsion angle of $173.19(12)^{\circ}$, which indicates that the molecule in the $\mathrm{Wu}$ et al. polymorph is almost planar (r.m.s. deviation $=0.031 \AA$ for 15 non-hydrogen atoms). The densities of (I) $\left[\rho=1.438 \mathrm{~g} \mathrm{~cm}^{-1}\right]$ and the Wu polymorph $[\rho=$ $\left.1.437 \mathrm{~g} \mathrm{~cm}^{-1}\right]$ are essentially identical.

There is one molecule in the asymmetric unit of (II), which crystallizes in space group $P \overline{1}$, as shown in Fig. 2. The C9/O1/ $\mathrm{O} 2$ grouping is almost coplanar with the mean-plane of the indole ring system (r.m.s. deviation $=0.009 \AA$ ), as indicated by the dihedral angle of $3.95(7)^{\circ}$ between $\mathrm{C} 1-\mathrm{C} 8 / \mathrm{N} 1$ and $\mathrm{C} 9 / \mathrm{O} 1 /$ O2. Atoms $\mathrm{Cl} 1$ and I1 deviate from the indole plane by -0.106 (2) and 0.081 (2) $\AA$, respectively. The conformation of the $\mathrm{C} 8-\mathrm{C} 9-\mathrm{O} 1-\mathrm{C} 10$ bond in (II) [torsion angle = $-177.42(16)^{\circ}$ ] is almost the same as the equivalent grouping in (I), but the $\mathrm{C} 9-\mathrm{O} 1-\mathrm{C} 10-\mathrm{C} 11$ torsion angle of $-178.33(17)^{\circ}$ is quite different, and indeed, the complete molecule of (II) is almost planar (r.m.s. deviation $=0.033 \AA$ for 16 non-hydrogen atoms).

\section{Supramolecular features}

In the crystal of (I), inversion dimers linked by pairs of $\mathrm{N}-$ $\mathrm{H} \cdots \mathrm{O}^{\mathrm{i}}$ [symmetry code: (i) $1-x, 2-y, 1-z$ ] hydrogen bonds (Table 1, Fig. 1) generate $R_{2}^{2}(10)$ loops. The first weak interaction to consider is aromatic $\pi-\pi$ stacking between the

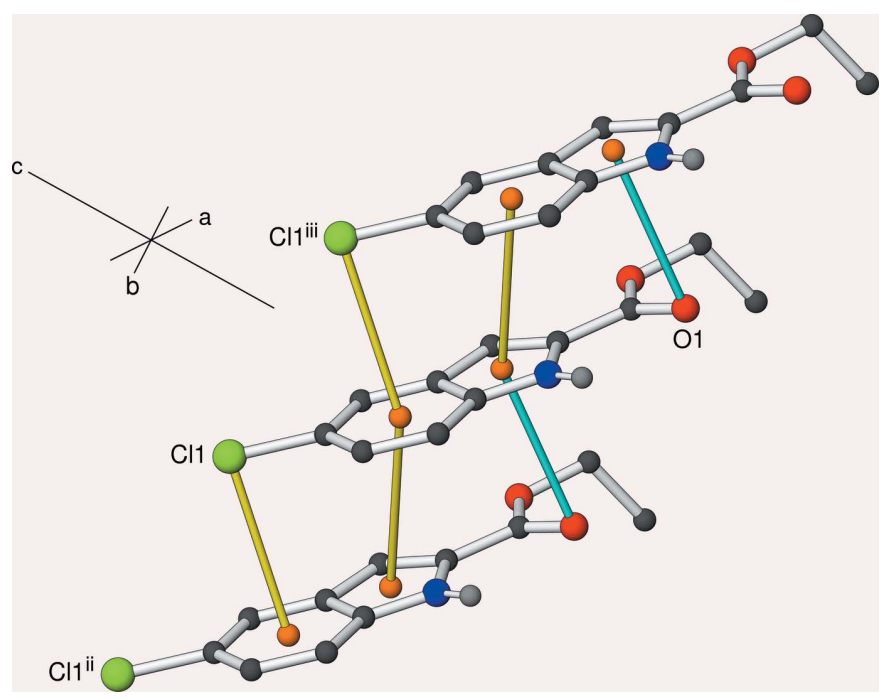

Figure 3

Partial packing diagram for (I), showing the formation of [010] chains linked by $\pi-\pi$ and $\mathrm{C}-\mathrm{Cl} \cdots \pi$ interactions (yellow lines). The long $\mathrm{C}=\mathrm{O} \cdots \pi$ contact is indicated by a cyan line. All hydrogen atoms except $\mathrm{H} 1$ are omitted for clarity. Symmetry codes (ii) $x, y+1, z$; (iii) $x, y-1, z$. 
Table 2

Hydrogen-bond geometry $\left(\AA,^{\circ}\right)$ for (II).

\begin{tabular}{lllll}
\hline$D-\mathrm{H} \cdots A$ & $D-\mathrm{H}$ & $\mathrm{H} \cdots A$ & $D \cdots A$ & $D-\mathrm{H} \cdots A$ \\
\hline $\mathrm{N} 1-\mathrm{H} 1 \cdots \mathrm{O} 2^{\mathrm{i}}$ & $0.77(3)$ & $2.06(3)$ & $2.821(2)$ & $168(3)$ \\
\hline
\end{tabular}

Symmetry code: (i) $-x,-y+2,-z+1$.

$\mathrm{C} 1-\mathrm{C} 6\left(\pi_{6}\right)$ ring and the $\mathrm{C} 1 / \mathrm{C} 6 / \mathrm{C} 7 / \mathrm{C} 8 / \mathrm{N} 1\left(\pi_{5}\right)$ five-membered ring displaced by translation in the $b$-axis direction (Fig. 3). The $\pi_{6}-\pi_{5}{ }^{\text {ii }}$ [symmetry code: (ii) $x, 1+y, z$ ] centroid-centroid separation is 3.7668 (9) $\AA$ and the inter-plane angle is $1.30(7)^{\circ}$. This interaction appears to be reinforced by a weak $\mathrm{C}-\mathrm{Cl} \cdots \pi_{5}{ }^{\mathrm{ii}}$ bond (Chifotides \& Dunbar, 2013); the chlorine atom lies almost directly above the centre of the sixmembered ring displaced in [010] with $\mathrm{Cl} \cdots \pi=3.5363$ (7) $\AA$ and $\mathrm{C}-\mathrm{Cl} \cdots \pi=86.35(5)^{\circ}$. This is very slightly shorter than the contact distance of $3.55 \AA$ for a chlorine atom and a benzene ring, assuming a radius of $1.75 \AA$ for $\mathrm{Cl}$ and a halfthickness of $1.8 \AA$ for a benzene ring. Thus, each benzene ring faces a chlorine atom on one face and a five-membered ring on the other $\left(\mathrm{Cl} \cdots \pi_{6} \cdots \pi_{5}=154.5^{\circ}\right)$. The carbonyl oxygen atom $(\mathrm{O} 2)$ of the ester group lies in a reasonable orientation to partake in a $\mathrm{C}=\mathrm{O} \cdots \pi_{5}$ bond (Gao et al., 2009) but here the $\mathrm{O} \cdots \pi_{5}^{\text {iii }}$ [symmetry code: (iii) $\left.x, y-1, z\right]$ separation of 3.4068 (11) $\AA$ is significantly greater than the van der Waals' radius sum of $3.32 \AA\left[\mathrm{C}=\mathrm{O} \cdots \pi_{5}=88.40(8)^{\circ}\right.$ and $\mathrm{O} \cdots \pi_{5} \cdots \pi_{6}$ $=153.9^{\circ} \mathrm{J}$ and can hardly be considered to be a bond. Taken together, the strong $(\mathrm{N}-\mathrm{H} \cdots \mathrm{O})$ and weak $(\pi-\pi, \mathrm{Cl} \cdots \pi)$ bonds lead to [010] double chains in the extended structure of (I).

Despite the fact that (I) and the Wu et al. (2013) polymorph of the same phase crystallize in the same space group, their

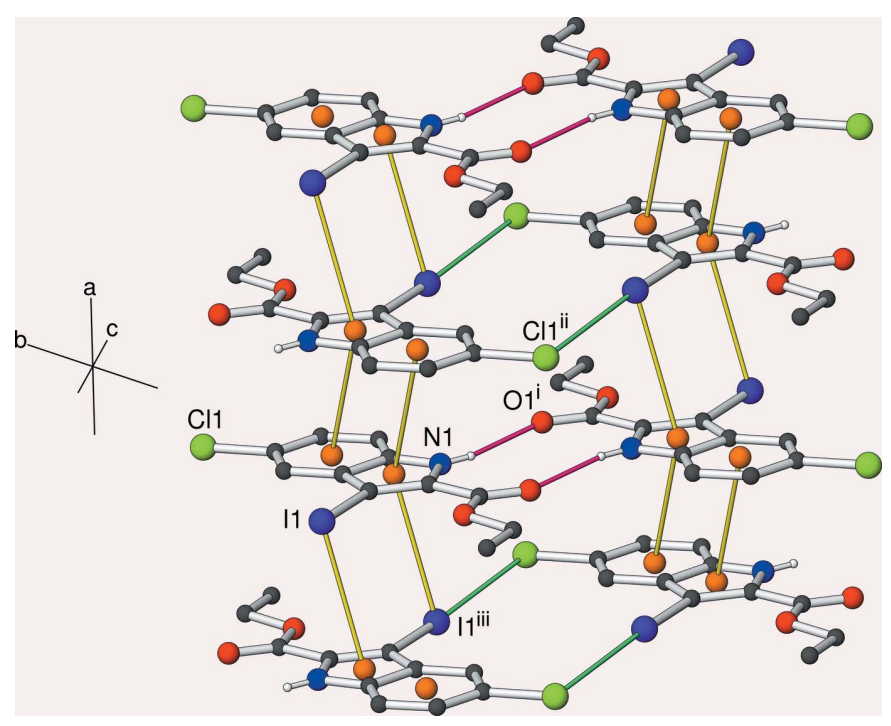

Figure 4

Partial packing diagram for (II), showing part of an (001) sheet. N$\mathrm{H} \cdots \mathrm{O}$ hydrogen bonds are indicated by crimson lines, $\pi-\pi$ and $\mathrm{I} \cdots \pi$ interactions by yellow lines and $\mathrm{I} \cdots \mathrm{Cl}$ halogen bonds by green lines. All hydrogen atoms except $\mathrm{H} 1$ are omitted for clarity. Symmetry codes (i) $-x, 2-y, 1-z$; (ii) $-x, 1-y, 1-z$; (iii) $1-x, 1-y, 1-z$. packing motifs are completely different. In the Wu phase, inversion dimers linked by pairs of $\mathrm{N}-\mathrm{H} \cdots \mathrm{O}$ hydrogen bonds also occur but there is no aromatic $\pi-\pi$ stacking (the shortest centroid-centroid separation is greater than $4.75 \AA$ ) and no $\mathrm{C}-\mathrm{Cl} \cdots \pi$ contacts. The only significant interaction indicated by a PLATON (Spek, 2009) analysis of the structure is a weak $\mathrm{C}-\mathrm{H} \cdots \pi_{5}$ bond $(\mathrm{H} \cdots \pi=2.72 \AA)$. Considered by itself, this interaction links the molecules into [010] chains; taken together, the $\mathrm{N}-\mathrm{H} \cdots \mathrm{O}$ and $\mathrm{C}-\mathrm{H} \cdots \pi$ interactions generate (110) sheets.

The crystal of (II) also features inversion dimers linked by pairs of $\mathrm{N}-\mathrm{H} \cdots \mathrm{O}^{\mathrm{i}}$ [symmetry code: (i) $-x, 2-y, 1-z$ ] hydrogen bonds (Table 2, Fig. 2) involving the equivalent atoms to (I) with the same graph-set motif. Aromatic $\pi-\pi$ stacking also occurs in the crystal of (II), but this time the molecules are related by inversion, rather than translation, symmetry: this operation 'flips' one of the molecules such that the six-membered ring in each molecule overlaps the fivemembered ring in the other (Fig. 6): the $\pi_{6}-\pi_{5}{ }^{\mathrm{ii}}$ [symmetry code: (ii) $-x, 1-y, 1-z$ ] separation of the centroids of the six- and five-membered rings is 3.6365 (14) $\AA$ and the interplanar angle is $0.92(13)^{\circ}$. The iodine atom of a molecule displaced in the [100] direction lies above the inversiongenerated five-membered ring to form a $\mathrm{C}-\mathrm{I} \cdots \pi_{5}$ bond with I $1 \cdots \pi_{5}$ iii [symmetry code: (iii) $1-x, 1-y, 1-z$ ] $=$ 3.6543 (11) $\AA$ and $\mathrm{C} 7-\mathrm{I} 1 \cdots \pi_{5}{ }^{\mathrm{iii}}=87.00$ (7) ${ }^{\circ}$. Thus, the fivemembered ring faces a six-membered ring on one face and an $\mathrm{I}$ atom on the other $\left(\mathrm{I} \cdots \pi_{5} \cdots \pi_{6}=148.6^{\circ}\right)$. The I atom also participates in a halogen bond (Desiraju et al., 2013) to the chlorine atom of an inversion-related molecule with $\mathrm{I} 1 \cdots \mathrm{Cl} 1^{\text {iv }}$ [symmetry code: (iv) $1-x,-y, 1-z$ ] $=3.6477$ (6) $\AA$ (van der Waals contact distance $=3.73 \AA), \mathrm{C} 7-\mathrm{I} 1 \cdots \mathrm{Cl} 1^{\text {iv }}=173.28(5)^{\circ}$ and $\mathrm{C}^{\text {iv }}-\mathrm{Cl}^{\text {iv }} \cdots \mathrm{I} 1=104.34(5)^{\circ}$. These angles clearly define this interaction as a type-II halogen bond (Pedireddi et al., 1994). Taken together, the weak and strong interactions lead to (001) sheets, with the centrosymmetric pairs of $\mathrm{I} \cdots \mathrm{Cl}$ halogen bonds and pairs of $\mathrm{N}-\mathrm{H} \cdots \mathrm{O}$ hydrogen bonds alternating with respect to the [100] direction (Fig. 4).

\section{Database survey}

A search of the Cambridge Structural Database (CSD; Groom et al., 2016) revealed 24 indole derivatives with an ester group at the 2-position of the ring system. In terms of halogen substitution, there were 58 5-chloro and just two 3-iodo derivatives. As noted above, VIHMUW (Wu et al., 2013) is a polymorph of (I): crystals of this phase in the form of colourless prisms were obtained by recrystallization from ethanol solution at room temperature, compared to colourless needles obtained from methanol solution at room temperature in the present study.

There has recently been debate on the significance - or otherwise - of weak intermolecular interactions in establishing the packing in molecular crystals (Dunitz, 2015; Thakur et al., 2015). The latter authors mentioned the role of weak interactions in establishing the structures of polymorphs and it is 
Table 3

Experimental details.

(I)

Crystal data

Chemical formula

$M_{\mathrm{r}}$

Crystal system, space group

Temperature (K)

$a, b, c(\AA)$

$\alpha, \beta, \gamma\left({ }^{\circ}\right)$

$V\left(\AA^{3}\right)$

$Z$

Radiation type

$\mu\left(\mathrm{mm}^{-1}\right)$

Crystal size $(\mathrm{mm})$

Data collection

Diffractometer

Absorption correction

$T_{\min }, T_{\max }$

No. of measured, independent and observed

$[I>2 \sigma(I)]$ reflections

$R_{\text {int }}$

$(\sin \theta / \lambda)_{\max }\left(\AA^{-1}\right)$

Refinemen

$R\left[F^{2}>2 \sigma\left(F^{2}\right)\right], w R\left(F^{2}\right), S$

No. of reflections

No. of parameters

$\mathrm{H}$-atom treatment

$\Delta \rho_{\max }, \Delta \rho_{\min }\left(\mathrm{e} \AA^{-3}\right)$

(II)

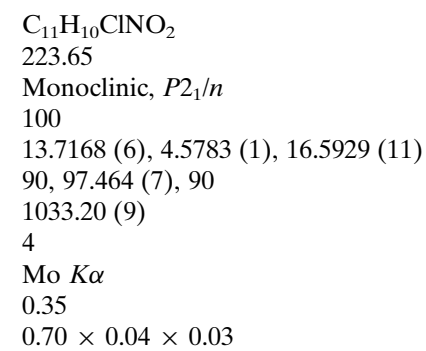

$0.70 \times 0.04 \times 0.03$

Rigaku Mercury CCD

Multi-scan (CrystalClear; Rigaku, 2012)

$0.793,0.990$

$7035,2340,2051$

0.022

0.649

$0.031,0.082,1.09$

2340

139

$\mathrm{H}$ atoms treated by a mixture of independent and constrained refinement $0.33,-0.23$

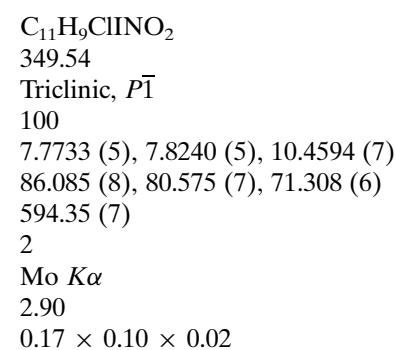

Rigaku Mercury CCD

Multi-scan (CrystalClear; Rigaku, 2012)

$0.638,0.944$

$7837,2739,2640$

0.031

0.650

$0.022,0.058,1.04$

2739

149

$\mathrm{H}$ atoms treated by a mixture of independent and constrained refinement $1.18,-0.44$

Computer programs: CrystalClear (Rigaku, 2012), SHELXS97 (Sheldrick, 2008), SHELXL2014 (Sheldrick, 2015), ORTEP-3 for Windows (Farrugia, 2012), ATOMS (Dowty, 2006) and publCIF (Westrip, 2010).

striking to us how different the packing motifs of (I) and VIHMUW are.

\section{Synthesis and crystallization}

To prepare (I), a mixture of ethyl 2-(2-[4-chlorophenyl]hydrazono)propanoate $(2.29 \mathrm{~g}, 9.51 \mathrm{mmol})$, prepared from p-chlorophenylhydrazine hydrochloride and ethyl pyruvate according to a published method (Zhang et al., 2011) and PPA $(22.54 \mathrm{~g})$ were refluxed in toluene $(40 \mathrm{ml})$ for $3 \mathrm{~h}$. After cooling, the solvent was decanted off and the solid residue was washed with toluene $(3 \times 50 \mathrm{ml})$. Evaporation of the combined organic phases under reduced pressure gave a yellow solid, flash chromatography of which (1:6 ethyl acetate, hexanes) afforded ethyl 5-chloro- $1 H$-indole-2-carboxylate as a yellow solid $(1.34 \mathrm{~g}, 63 \%)$. Colourless needles of (I) were recrystallized from methanol solution at room temperature. $\delta \mathrm{C}\left(101 \mathrm{MHz} ; \mathrm{CDCl}_{3}\right) 162.0(\mathrm{Cq}), 135.2(\mathrm{Cq}), 128.9(\mathrm{Cq}), 128.6$ $(\mathrm{Cq}), 126.7(\mathrm{Cq}), 126.0(\mathrm{CH}), 121.9(\mathrm{CH}), 113.1(\mathrm{CH}), 108.1$ $(\mathrm{CH}), 61.5\left(\mathrm{CH}_{2}\right)$ and $14.5\left(\mathrm{CH}_{3}\right) ; \delta \mathrm{H}\left(400 \mathrm{MHz} ; \mathrm{CDCl}_{3}\right) 8.91$ $(1 \mathrm{H}, b r s), 7.67(1 \mathrm{H}, s), 7.35-7.28(2 \mathrm{H}, m), 7.15(1 \mathrm{H}, s), 4.41$ $\left(2 \mathrm{H}, q, J\right.$ 7.1) and $1.41\left(3 \mathrm{H}, t, J\right.$ 7.1); $R_{\mathrm{f}} 0.29$ (1:6 EtOAc, hexanes); m.p. 440-441 K; IR (Nujol, $\mathrm{cm}^{-1}$ ) 3310, 1728, 1697, 1264, 1080 and 877; HRMS (ESI) for $\mathrm{C}_{11} \mathrm{H}_{11}{ }^{35} \mathrm{ClNO}_{2}[M+\mathrm{H}]^{+}$ calculated 224.0479, found 224.0466.

To prepare (II), potassium hydroxide $(1.804 \mathrm{~g}, 32.2 \mathrm{mmol})$ was added to a solution of (I) (1.215 g, $5.43 \mathrm{mmol})$ in dry DMF
$(6.0 \mathrm{ml})$ at $273 \mathrm{~K}$ and stirred for $10 \mathrm{~min}$. Separately, a solution of iodine $(1.710 \mathrm{~g}, 6.74 \mathrm{mmol})$ in dry DMF $(6.75 \mathrm{ml})$ was prepared. The two liquids were combined and stirred over ice for $90 \mathrm{~min}$. Pouring the reaction mixture into a saturated aqueous solution of ammonium chloride and sodium thiosulfate $(60 \mathrm{ml})$ precipitated a brown solid. This was collected by filtration and purified by flash chromatography (1:8 ethyl acetate, hexanes) to afford ethyl 5-chloro-3-iodo- $1 H$-indole-2carboxylate as a yellow solid $(1.825 \mathrm{~g}, 80 \%)$. Pale-yellow plates of (II) were recrystallized from methanol solution at room temperature. $\delta \mathrm{C}\left(101 \mathrm{MHz}\right.$; DMSO- $\left.d_{6}\right) 160.5(\mathrm{Cq}), 135.8$ $(\mathrm{Cq}), 132.1(\mathrm{Cq}), 128.9(\mathrm{Cq}), 126.5(\mathrm{CH}), 126.3(\mathrm{Cq}), 121.8$ $(\mathrm{CH}), 115.4(\mathrm{CH}), 65.2(\mathrm{Cq}), 61.4\left(\mathrm{CH}_{2}\right)$ and $14.6\left(\mathrm{CH}_{3}\right)$; $\delta \mathrm{H}\left(400 \mathrm{MHz} ; \mathrm{DMSO}-d_{6}\right) 12.42(1 \mathrm{H}, b r s), 7.47(1 \mathrm{H}, d, J$ 8.4), $7.39(1 \mathrm{H}, d, J 1.6), 7.31(1 \mathrm{H}, d d, J 2.0,9.2), 4.36(2 \mathrm{H}, q, J 7.2)$ and $1.36(3 \mathrm{H}, t, J 7.0) ; R_{\mathrm{f}} 0.13$ (1:8 ethyl acetate, hexanes); m.p. $412 \mathrm{~K}$, IR (KBr, cm ${ }^{-1}$ ) 3291, 2977, 1744, 1683, 1514, 1332, 1115, 1080, 772, 749 and 604; HRMS (ESI) for $\mathrm{C}_{11} \mathrm{H}_{10}{ }^{35} \mathrm{ClINO}_{2}[M+\mathrm{H}]^{+}$calculated 349.9445 , found 349.9453.

\section{Refinement}

Crystal data, data collection and structure refinement details are summarized in Table 3. The $\mathrm{N}$-bound $\mathrm{H}$ atoms were located in difference maps and their positions freely refined. The C-bound $\mathrm{H}$ atoms were placed geometrically $(\mathrm{C}-\mathrm{H}=$ 
0.93-0.98 $\AA$ ) and refined as riding atoms. The constraint $U_{\text {iso }}(\mathrm{H})=1.2 U_{\text {eq }}($ carrier $)$ or $1.5 U_{\text {eq }}$ (methyl carrier) was applied in all cases. The $-\mathrm{CH}_{3}$ groups were allowed to rotate, but not to tip, to best fit the electron density.

\section{Acknowledgements}

We thank the EPSRC National Crystallography Service (University of Southampton) for the data collections and the EPSRC National Mass Spectrometry Service (University of Swansea) for the HRMS data.

\section{References}

Chifotides, H. T. \& Dunbar, K. R. (2013). Acc. Chem. Res. 46, 894906.

Desiraju, G. R., Ho, P. S., Kloo, L., Legon, A. C., Marquardt, R., Metrangolo, P., Politzer, P., Resnati, G. \& Rissanen, K. (2013). Pure Appl. Chem. 85, 1711-1713.

Dowty, E. W. (2006). ATOMS. Shape Software, Kingsport, Tennessee, USA.
Dunitz, J. D. (2015). IUCrJ, 2, 157-158.

Farrugia, L. J. (2012). J. Appl. Cryst. 45, 849-854.

Gao, X.-L., Lu, L.-P. \& Zhu, M.-L. (2009). Acta Cryst. C65, o1230127.

Groom, C. R., Bruno, I. J., Lightfoot, M. P. \& Ward, S. C. (2016). Acta Cryst. B72, 171-179.

Kerr, J. R. (2013). PhD Thesis 'Allosteric modulation of the CB1 receptor'. University of Aberdeen.

Kerr, J. R., Trembleau, L., Storey, J. M. D., Wardell, J. L. \& Harrison, W. T. A. (2016). Acta Cryst. E72, 699-703.

Pedireddi, V. R., Reddy, D. S., Goud, B. S., Craig, D. C., Rae, A. D. \& Desiraju, G. R. (1994). J. Chem. Soc. Perkin Trans. 2, pp. 23532360.

Rigaku (2012). CrystalClear. Rigaku Corporation, Tokyo, Japan.

Sheldrick, G. M. (2008). Acta Cryst. A64, 112-122.

Sheldrick, G. M. (2015). Acta Cryst. C71, 3-8.

Spek, A. L. (2009). Acta Cryst. D65, 148-155.

Thakur, T. S., Dubey, R. \& Desiraju, G. R. (2015). IUCrJ, 2, 159-160.

Westrip, S. P. (2010). J. Appl. Cryst. 43, 920-925.

Wu, J., Liu, Y.-L., Wu, H., Wang, H.-Y. \& Zou, P. (2013). Z. Krist. New Cryst. Struct. 228, 185-186.

Zhang, F., Zhao, Y., Sun, L., Ding, L., Gu, Y. \& Gong, P. (2011). Eur. J. Med. Chem. 46, 3149-3157. 


\section{supporting information}

Acta Cryst. (2016). E72, 964-968 [https://doi.org/10.1107/S2056989016008616]

Weak interactions in the crystal structures of two indole derivatives

Jamie R. Kerr, Laurent Trembleau, John M. D. Storey, James L. Wardell and William T. A.

Harrison

Computing details

For both compounds, data collection: CrystalClear (Rigaku, 2012); cell refinement: CrystalClear (Rigaku, 2012); data reduction: CrystalClear (Rigaku, 2012); program(s) used to solve structure: SHELXS97 (Sheldrick, 2008); program(s) used to refine structure: SHELXL2014 (Sheldrick, 2015); molecular graphics: ORTEP-3 for Windows (Farrugia, 2012) and ATOMS (Dowty, 2006); software used to prepare material for publication: publCIF (Westrip, 2010).

(I) Ethyl 5-chloro-1H-indole-2-carboxylate

Crystal data

$\mathrm{C}_{11} \mathrm{H}_{10} \mathrm{ClNO}_{2}$

$M_{r}=223.65$

Monoclinic, $P 2_{1} / n$

$a=13.7168(6) \AA$

$b=4.5783(1) \AA$

$c=16.5929(11) \AA$

$\beta=97.464(7)^{\circ}$

$V=1033.20(9) \AA^{3}$

$Z=4$

Data collection

Rigaku Mercury CCD diffractometer

$\omega$ scans

Absorption correction: multi-scan

(CrystalClear; Rigaku, 2012)

$T_{\min }=0.793, T_{\max }=0.990$

7035 measured reflections

\section{Refinement}

Refinement on $F^{2}$

Least-squares matrix: full

$R\left[F^{2}>2 \sigma\left(F^{2}\right)\right]=0.031$

$w R\left(F^{2}\right)=0.082$

$S=1.09$

2340 reflections

139 parameters

0 restraints
$F(000)=464$

$D_{\mathrm{x}}=1.438 \mathrm{Mg} \mathrm{m}^{-3}$

Mo $K \alpha$ radiation, $\lambda=0.71073 \AA$

Cell parameters from 6396 reflections

$\theta=3.0-27.5^{\circ}$

$\mu=0.35 \mathrm{~mm}^{-1}$

$T=100 \mathrm{~K}$

Rod, colourless

$0.70 \times 0.04 \times 0.03 \mathrm{~mm}$

2340 independent reflections

2051 reflections with $I>2 \sigma(I)$

$R_{\text {int }}=0.022$

$\theta_{\max }=27.5^{\circ}, \theta_{\min }=3.0^{\circ}$

$h=-17 \rightarrow 17$

$k=-4 \rightarrow 5$

$l=-19 \rightarrow 21$

Primary atom site location: structure-invariant direct methods

Hydrogen site location: mixed

$\mathrm{H}$ atoms treated by a mixture of independent and constrained refinement

$w=1 /\left[\sigma^{2}\left(F_{\mathrm{o}}^{2}\right)+(0.0408 P)^{2}+0.4177 P\right]$

where $P=\left(F_{\mathrm{o}}{ }^{2}+2 F_{\mathrm{c}}{ }^{2}\right) / 3$

$(\Delta / \sigma)_{\max }<0.001$

$\Delta \rho_{\max }=0.33 \mathrm{e} \AA^{-3}$

$\Delta \rho_{\min }=-0.23$ e $\AA^{-3}$ 


\section{Special details}

Geometry. All esds (except the esd in the dihedral angle between two 1.s. planes) are estimated using the full covariance matrix. The cell esds are taken into account individually in the estimation of esds in distances, angles and torsion angles; correlations between esds in cell parameters are only used when they are defined by crystal symmetry. An approximate (isotropic) treatment of cell esds is used for estimating esds involving l.s. planes.

Fractional atomic coordinates and isotropic or equivalent isotropic displacement parameters $\left(\hat{A}^{2}\right)$

\begin{tabular}{lllll}
\hline & $x$ & $y$ & $z$ & $U_{\text {iso }} * / U_{\text {eq }}$ \\
\hline C1 & $0.52594(9)$ & $0.5040(3)$ & $0.35005(8)$ & $0.0142(3)$ \\
C2 & $0.62255(10)$ & $0.4033(3)$ & $0.35004(8)$ & $0.0176(3)$ \\
H2 & 0.6742 & 0.4669 & 0.3899 & $0.021^{*}$ \\
C3 & $0.63988(9)$ & $0.2090(3)$ & $0.29013(8)$ & $0.0171(3)$ \\
H3 & 0.7044 & 0.1360 & 0.2883 & $0.021^{*}$ \\
C4 & $0.56188(10)$ & $0.1181(3)$ & $0.23127(8)$ & $0.0153(3)$ \\
C5 & $0.46670(9)$ & $0.2127(3)$ & $0.23045(8)$ & $0.0146(3)$ \\
H5 & 0.4157 & 0.1459 & 0.1905 & $0.017^{*}$ \\
C6 & $0.44761(9)$ & $0.4121(3)$ & $0.29091(8)$ & $0.0134(3)$ \\
C7 & $0.36151(9)$ & $0.5605(3)$ & $0.30827(8)$ & $0.0136(3)$ \\
H7 & 0.2972 & 0.5434 & 0.2795 & $0.016^{*}$ \\
C8 & $0.38999(9)$ & $0.7341(3)$ & $0.37519(7)$ & $0.0135(3)$ \\
C9 & $0.33337(9)$ & $0.9388(3)$ & $0.41806(7)$ & $0.0133(3)$ \\
C10 & $0.17639(10)$ & $1.1516(3)$ & $0.42258(8)$ & $0.0174(3)$ \\
H10A & 0.1186 & 1.2025 & 0.3829 & $0.021^{*}$ \\
H10B & 0.2134 & 1.3334 & 0.4376 & $0.021^{*}$ \\
C11 & $0.14202(11)$ & $1.0204(4)$ & $0.49746(9)$ & $0.0271(3)$ \\
H11A & 0.1002 & 1.1610 & 0.5214 & $0.041^{*}$ \\
H11B & 0.1044 & 0.8421 & 0.4825 & $0.041^{*}$ \\
H11C & 0.1991 & 0.9728 & 0.5371 & $0.041^{*}$ \\
N1 & $0.48871(8)$ & $0.6981(3)$ & $0.40047(7)$ & $0.0147(2)$ \\
H1 & $0.5235(12)$ & $0.789(4)$ & $0.4411(10)$ & $0.018^{*}$ \\
O1 & $0.23886(6)$ & $0.9493(2)$ & $0.38517(5)$ & $0.0152(2)$ \\
O2 & $0.36753(7)$ & $1.0841(2)$ & $0.47654(6)$ & $0.0184(2)$ \\
C11 & $0.58935(2)$ & $-0.12275(8)$ & $0.15557(2)$ & $0.01929(11)$ \\
& & & &
\end{tabular}

Atomic displacement parameters $\left(\AA^{2}\right)$

\begin{tabular}{lllllll}
\hline & $U^{11}$ & $U^{22}$ & $U^{33}$ & $U^{12}$ & $U^{13}$ & $U^{23}$ \\
\hline C1 & $0.0137(6)$ & $0.0138(6)$ & $0.0147(6)$ & $-0.0016(5)$ & $-0.0002(5)$ & $0.0003(5)$ \\
C2 & $0.0128(6)$ & $0.0196(7)$ & $0.0193(6)$ & $-0.0010(5)$ & $-0.0026(5)$ & $-0.0010(5)$ \\
C3 & $0.0123(6)$ & $0.0175(7)$ & $0.0213(7)$ & $0.0013(5)$ & $0.0012(5)$ & $0.0009(6)$ \\
C4 & $0.0175(6)$ & $0.0126(6)$ & $0.0164(6)$ & $-0.0001(5)$ & $0.0040(5)$ & $-0.0007(5)$ \\
C5 & $0.0144(6)$ & $0.0146(6)$ & $0.0140(6)$ & $-0.0017(5)$ & $-0.0008(5)$ & $0.0004(5)$ \\
C6 & $0.0134(6)$ & $0.0126(6)$ & $0.0135(6)$ & $-0.0014(5)$ & $-0.0007(5)$ & $0.0020(5)$ \\
C7 & $0.0130(6)$ & $0.0140(6)$ & $0.0134(6)$ & $-0.0011(5)$ & $-0.0004(4)$ & $0.0008(5)$ \\
C8 & $0.0122(6)$ & $0.0149(6)$ & $0.0129(6)$ & $-0.0012(5)$ & $-0.0007(4)$ & $0.0014(5)$ \\
C9 & $0.0142(6)$ & $0.0132(6)$ & $0.0122(6)$ & $-0.0014(5)$ & $0.0007(4)$ & $0.0025(5)$ \\
C10 & $0.0149(6)$ & $0.0169(7)$ & $0.0203(7)$ & $0.0043(5)$ & $0.0013(5)$ & $-0.0010(5)$
\end{tabular}




\begin{tabular}{lllllll}
$\mathrm{C} 11$ & $0.0248(7)$ & $0.0318(9)$ & $0.0265(8)$ & $0.0035(7)$ & $0.0106(6)$ & $0.0017(7)$ \\
$\mathrm{N} 1$ & $0.0125(5)$ & $0.0175(6)$ & $0.0130(5)$ & $-0.0004(5)$ & $-0.0023(4)$ & $-0.0027(4)$ \\
O1 & $0.0126(4)$ & $0.0171(5)$ & $0.0154(4)$ & $0.0015(4)$ & $-0.0005(3)$ & $-0.0023(4)$ \\
O2 & $0.0164(5)$ & $0.0218(5)$ & $0.0158(5)$ & $-0.0001(4)$ & $-0.0018(4)$ & $-0.0050(4)$ \\
C11 & $0.01786(17)$ & $0.01983(19)$ & $0.02054(18)$ & $0.00168(13)$ & $0.00388(12)$ & $-0.00493(13)$ \\
\hline
\end{tabular}

Geometric parameters $\left(\hat{A},{ }^{\circ}\right)$

\begin{tabular}{|c|c|c|c|}
\hline $\mathrm{C} 1-\mathrm{N} 1$ & $1.3644(18)$ & $\mathrm{C} 7-\mathrm{H} 7$ & 0.9500 \\
\hline $\mathrm{C} 1-\mathrm{C} 2$ & $1.4032(18)$ & $\mathrm{C} 8-\mathrm{N} 1$ & $1.3744(16)$ \\
\hline $\mathrm{C} 1-\mathrm{C} 6$ & $1.4215(17)$ & $\mathrm{C} 8-\mathrm{C} 9$ & $1.4599(19)$ \\
\hline $\mathrm{C} 2-\mathrm{C} 3$ & $1.3774(19)$ & $\mathrm{C} 9-\mathrm{O} 2$ & $1.2187(16)$ \\
\hline $\mathrm{C} 2-\mathrm{H} 2$ & 0.9500 & $\mathrm{C} 9-\mathrm{O} 1$ & $1.3405(15)$ \\
\hline $\mathrm{C} 3-\mathrm{C} 4$ & $1.4145(19)$ & $\mathrm{C} 10-\mathrm{O} 1$ & $1.4543(16)$ \\
\hline $\mathrm{C} 3-\mathrm{H} 3$ & 0.9500 & $\mathrm{C} 10-\mathrm{C} 11$ & $1.5098(19)$ \\
\hline $\mathrm{C} 4-\mathrm{C} 5$ & $1.3739(18)$ & $\mathrm{C} 10-\mathrm{H} 10 \mathrm{~A}$ & 0.9900 \\
\hline $\mathrm{C} 4-\mathrm{Cl1}$ & $1.7488(14)$ & $\mathrm{C} 10-\mathrm{H} 10 \mathrm{~B}$ & 0.9900 \\
\hline $\mathrm{C} 5-\mathrm{C} 6$ & $1.4059(18)$ & $\mathrm{C} 11-\mathrm{H} 11 \mathrm{~A}$ & 0.9800 \\
\hline $\mathrm{C} 5-\mathrm{H} 5$ & 0.9500 & C11-H11B & 0.9800 \\
\hline $\mathrm{C} 6-\mathrm{C} 7$ & $1.4240(18)$ & $\mathrm{C} 11-\mathrm{H} 11 \mathrm{C}$ & 0.9800 \\
\hline $\mathrm{C} 7-\mathrm{C} 8$ & $1.3800(18)$ & $\mathrm{N} 1-\mathrm{H} 1$ & $0.878(17)$ \\
\hline $\mathrm{N} 1-\mathrm{C} 1-\mathrm{C} 2$ & $130.00(12)$ & $\mathrm{N} 1-\mathrm{C} 8-\mathrm{C} 9$ & $119.57(11)$ \\
\hline $\mathrm{N} 1-\mathrm{C} 1-\mathrm{C} 6$ & $107.86(11)$ & $\mathrm{C} 7-\mathrm{C} 8-\mathrm{C} 9$ & $130.46(12)$ \\
\hline $\mathrm{C} 2-\mathrm{C} 1-\mathrm{C} 6$ & $122.13(13)$ & $\mathrm{O} 2-\mathrm{C} 9-\mathrm{O} 1$ & $123.78(12)$ \\
\hline $\mathrm{C} 3-\mathrm{C} 2-\mathrm{C} 1$ & $117.63(12)$ & $\mathrm{O} 2-\mathrm{C} 9-\mathrm{C} 8$ & $124.37(12)$ \\
\hline $\mathrm{C} 3-\mathrm{C} 2-\mathrm{H} 2$ & 121.2 & $\mathrm{O} 1-\mathrm{C} 9-\mathrm{C} 8$ & 111.85 \\
\hline $\mathrm{C} 1-\mathrm{C} 2-\mathrm{H} 2$ & 121.2 & $\mathrm{O} 1-\mathrm{C} 10-\mathrm{C} 11$ & $111.24(12)$ \\
\hline $\mathrm{C} 2-\mathrm{C} 3-\mathrm{C} 4$ & $120.17(12)$ & $\mathrm{O} 1-\mathrm{C} 10-\mathrm{H} 10 \mathrm{~A}$ & 109.4 \\
\hline $\mathrm{C} 2-\mathrm{C} 3-\mathrm{H} 3$ & 119.9 & $\mathrm{C} 11-\mathrm{C} 10-\mathrm{H} 10 \mathrm{~A}$ & 109.4 \\
\hline $\mathrm{C} 4-\mathrm{C} 3-\mathrm{H} 3$ & 119.9 & $\mathrm{O} 1-\mathrm{C} 10-\mathrm{H} 10 \mathrm{~B}$ & 109.4 \\
\hline $\mathrm{C} 5-\mathrm{C} 4-\mathrm{C} 3$ & $123.13(12)$ & $\mathrm{C} 11-\mathrm{C} 10-\mathrm{H} 10 \mathrm{~B}$ & 109.4 \\
\hline $\mathrm{C} 5-\mathrm{C} 4-\mathrm{Cl1}$ & $119.00(10)$ & $\mathrm{H} 10 \mathrm{~A}-\mathrm{C} 10-\mathrm{H} 10 \mathrm{~B}$ & 108.0 \\
\hline $\mathrm{C} 3-\mathrm{C} 4-\mathrm{Cl} 1$ & $117.87(10)$ & $\mathrm{C} 10-\mathrm{C} 11-\mathrm{H} 11 \mathrm{~A}$ & 109.5 \\
\hline $\mathrm{C} 4-\mathrm{C} 5-\mathrm{C} 6$ & $117.55(12)$ & $\mathrm{C} 10-\mathrm{C} 11-\mathrm{H} 11 \mathrm{~B}$ & 109.5 \\
\hline $\mathrm{C} 4-\mathrm{C} 5-\mathrm{H} 5$ & 121.2 & $\mathrm{H} 11 \mathrm{~A}-\mathrm{C} 11-\mathrm{H} 11 \mathrm{~B}$ & 109.5 \\
\hline $\mathrm{C} 6-\mathrm{C} 5-\mathrm{H} 5$ & 121.2 & $\mathrm{C} 10-\mathrm{C} 11-\mathrm{H} 11 \mathrm{C}$ & 109.5 \\
\hline $\mathrm{C} 5-\mathrm{C} 6-\mathrm{C} 1$ & $119.38(12)$ & $\mathrm{H} 11 \mathrm{~A}-\mathrm{C} 11-\mathrm{H} 11 \mathrm{C}$ & 109.5 \\
\hline $\mathrm{C} 5-\mathrm{C} 6-\mathrm{C} 7$ & $133.66(12)$ & $\mathrm{H} 11 \mathrm{~B}-\mathrm{C} 11-\mathrm{H} 11 \mathrm{C}$ & 109.5 \\
\hline $\mathrm{C} 1-\mathrm{C} 6-\mathrm{C} 7$ & $106.95(11)$ & $\mathrm{C} 1-\mathrm{N} 1-\mathrm{C} 8$ & $108.85(11)$ \\
\hline $\mathrm{C} 8-\mathrm{C} 7-\mathrm{C} 6$ & $106.38(11)$ & $\mathrm{C} 1-\mathrm{N} 1-\mathrm{H} 1$ & $124.7(11)$ \\
\hline $\mathrm{C} 8-\mathrm{C} 7-\mathrm{H} 7$ & 126.8 & $\mathrm{C} 8-\mathrm{N} 1-\mathrm{H} 1$ & $126.4(11)$ \\
\hline $\mathrm{C} 6-\mathrm{C} 7-\mathrm{H} 7$ & 126.8 & $\mathrm{C} 9-\mathrm{O} 1-\mathrm{C} 10$ & $116.19(10)$ \\
\hline $\mathrm{N} 1-\mathrm{C} 8-\mathrm{C} 7$ & $109.96(12)$ & & \\
\hline $\mathrm{N} 1-\mathrm{C} 1-\mathrm{C} 2-\mathrm{C} 3$ & $-178.66(14)$ & $\mathrm{C} 1-\mathrm{C} 6-\mathrm{C} 7-\mathrm{C} 8$ & $0.37(15)$ \\
\hline $\mathrm{C} 6-\mathrm{C} 1-\mathrm{C} 2-\mathrm{C} 3$ & $-0.1(2)$ & $\mathrm{C} 6-\mathrm{C} 7-\mathrm{C} 8-\mathrm{N} 1$ & $-0.63(15)$ \\
\hline $\mathrm{C} 1-\mathrm{C} 2-\mathrm{C} 3-\mathrm{C} 4$ & $0.2(2)$ & $\mathrm{C} 6-\mathrm{C} 7-\mathrm{C} 8-\mathrm{C} 9$ & $178.10(13)$ \\
\hline
\end{tabular}




$\begin{array}{llll}\mathrm{C} 2-\mathrm{C} 3-\mathrm{C} 4-\mathrm{C} 5 & -0.5(2) & \mathrm{N} 1-\mathrm{C} 8-\mathrm{C} 9-\mathrm{O} 2 & -0.4(2) \\ \mathrm{C} 2-\mathrm{C} 3-\mathrm{C} 4-\mathrm{C} 11 & 178.63(11) & \mathrm{C} 7-\mathrm{C} 8-\mathrm{C} 9-\mathrm{O} 2 & -178.99(14) \\ \mathrm{C} 3-\mathrm{C} 4-\mathrm{C} 5-\mathrm{C} 6 & 0.8(2) & \mathrm{N} 1-\mathrm{C} 8-\mathrm{C} 9-\mathrm{O} 1 & 179.38(11) \\ \mathrm{C} 11-\mathrm{C} 4-\mathrm{C} 5-\mathrm{C} 6 & -178.38(10) & \mathrm{C} 7-\mathrm{C} 8-\mathrm{C} 9-\mathrm{O} 1 & 0.8(2) \\ \mathrm{C} 4-\mathrm{C} 5-\mathrm{C} 6-\mathrm{C} 1 & -0.67(19) & \mathrm{C} 2-\mathrm{C} 1-\mathrm{N} 1-\mathrm{C} 8 & 178.35(14) \\ \mathrm{C} 4-\mathrm{C} 5-\mathrm{C} 6-\mathrm{C} 7 & 178.26(14) & \mathrm{C} 6-\mathrm{C} 1-\mathrm{N} 1-\mathrm{C} 8 & -0.40(15) \\ \mathrm{N} 1-\mathrm{C} 1-\mathrm{C} 6-\mathrm{C} 5 & 179.20(11) & \mathrm{C} 7-\mathrm{C} 8-\mathrm{N} 1-\mathrm{C} 1 & 0.65(15) \\ \mathrm{C} 2-\mathrm{C} 1-\mathrm{C} 6-\mathrm{C} 5 & 0.3(2) & \mathrm{C} 9-\mathrm{C} 8-\mathrm{N} 1-\mathrm{C} 1 & -178.23(12) \\ \mathrm{N} 1-\mathrm{C} 1-\mathrm{C} 6-\mathrm{C} 7 & 0.01(15) & \mathrm{O} 2-\mathrm{C} 9-\mathrm{O} 1-\mathrm{C} 10 & 0.89(18) \\ \mathrm{C} 2-\mathrm{C} 1-\mathrm{C} 6-\mathrm{C} 7 & -178.85(12) & \mathrm{C} 8-\mathrm{C} 9-\mathrm{O} 1-\mathrm{C} 10 & -178.86(11) \\ \mathrm{C} 5-\mathrm{C} 6-\mathrm{C} 7-\mathrm{C} 8 & -178.65(14) & \mathrm{C} 11-\mathrm{C} 10-\mathrm{O} 1-\mathrm{C} 9 & -81.73(14)\end{array}$

Hydrogen-bond geometry $\left(A,{ }^{\circ}\right)$

\begin{tabular}{lllll}
\hline$D-\mathrm{H} \cdots A$ & $D-\mathrm{H}$ & $\mathrm{H} \cdots A$ & $D \cdots A$ & $D-\mathrm{H} \cdots A$ \\
\hline $\mathrm{N} 1-\mathrm{H} 1 \cdots \mathrm{O} 2^{\mathrm{i}}$ & $0.878(17)$ & $1.977(17)$ & $2.8288(15)$ & $163.0(15)$ \\
\hline
\end{tabular}

Symmetry code: (i) $-x+1,-y+2,-z+1$.

(II) Ethyl 5-chloro-3-iodo-1 $\mathrm{H}$-indole-2-carboxylate

Crystal data

$\mathrm{C}_{11} \mathrm{H}_{9} \mathrm{ClINO}_{2}$

$M_{r}=349.54$

Triclinic, $P \overline{1}$

$a=7.7733(5) \AA$

$b=7.8240(5) \AA$

$c=10.4594(7) \AA$

$\alpha=86.085(8)^{\circ}$

$\beta=80.575(7)^{\circ}$

$\gamma=71.308(6)^{\circ}$

$V=594.35(7) \AA^{3}$

\section{Data collection}

Rigaku Mercury CCD diffractometer

$\omega$ scans

Absorption correction: multi-scan

(CrystalClear; Rigaku, 2012)

$T_{\min }=0.638, T_{\max }=0.944$

7837 measured reflections

\section{Refinement}

Refinement on $F^{2}$

Least-squares matrix: full

$R\left[F^{2}>2 \sigma\left(F^{2}\right)\right]=0.022$

$w R\left(F^{2}\right)=0.058$

$S=1.04$

2739 reflections

149 parameters

0 restraints
$Z=2$

$F(000)=336$

$D_{\mathrm{x}}=1.953 \mathrm{Mg} \mathrm{m}^{-3}$

Mo $K \alpha$ radiation, $\lambda=0.71073 \AA$

Cell parameters from 7985 reflections

$\theta=2.7-27.5^{\circ}$

$\mu=2.90 \mathrm{~mm}^{-1}$

$T=100 \mathrm{~K}$

Plate, colourless

$0.17 \times 0.10 \times 0.02 \mathrm{~mm}$

2739 independent reflections

2640 reflections with $I>2 \sigma(I)$

$R_{\text {int }}=0.031$

$\theta_{\text {max }}=27.5^{\circ}, \theta_{\text {min }}=2.8^{\circ}$

$h=-8 \rightarrow 10$

$k=-10 \rightarrow 10$

$l=-12 \rightarrow 13$

Hydrogen site location: mixed

$\mathrm{H}$ atoms treated by a mixture of independent and constrained refinement

$w=1 /\left[\sigma^{2}\left(F_{\mathrm{o}}^{2}\right)+(0.0409 P)^{2}+0.1325 P\right]$

where $P=\left(F_{\mathrm{o}}^{2}+2 F_{\mathrm{c}}^{2}\right) / 3$

$(\Delta / \sigma)_{\max }=0.002$

$\Delta \rho_{\max }=1.18 \mathrm{e} \AA^{-3}$

$\Delta \rho_{\min }=-0.44$ e $\AA^{-3}$ 


\section{Special details}

Geometry. All esds (except the esd in the dihedral angle between two 1.s. planes) are estimated using the full covariance matrix. The cell esds are taken into account individually in the estimation of esds in distances, angles and torsion angles; correlations between esds in cell parameters are only used when they are defined by crystal symmetry. An approximate (isotropic) treatment of cell esds is used for estimating esds involving l.s. planes.

Fractional atomic coordinates and isotropic or equivalent isotropic displacement parameters $\left(\hat{A}^{2}\right)$

\begin{tabular}{lllll}
\hline & $x$ & $y$ & $z$ & $U_{\text {iso }} * / U_{\text {eq }}$ \\
\hline C1 & $0.0847(3)$ & $0.5746(3)$ & $0.6457(2)$ & $0.0165(4)$ \\
C2 & $-0.0166(3)$ & $0.5738(3)$ & $0.7698(2)$ & $0.0190(4)$ \\
H2 & -0.0956 & 0.6824 & 0.8097 & $0.023^{*}$ \\
C3 & $0.0037(3)$ & $0.4078(3)$ & $0.8316(2)$ & $0.0204(4)$ \\
H3 & -0.0631 & 0.4013 & 0.9154 & $0.024^{*}$ \\
C4 & $0.1227(3)$ & $0.2486(3)$ & $0.7712(2)$ & $0.0185(4)$ \\
C5 & $0.2249(3)$ & $0.2468(3)$ & $0.6505(2)$ & $0.0176(4)$ \\
H5 & 0.3045 & 0.1374 & 0.6122 & $0.021^{*}$ \\
C6 & $0.2064(3)$ & $0.4142(3)$ & $0.5863(2)$ & $0.0160(4)$ \\
C7 & $0.2860(3)$ & $0.4665(3)$ & $0.46397(19)$ & $0.0144(4)$ \\
C8 & $0.2117(3)$ & $0.6518(3)$ & $0.45207(19)$ & $0.0155(4)$ \\
C9 & $0.2427(3)$ & $0.7849(3)$ & $0.3534(2)$ & $0.0162(4)$ \\
C10 & $0.4005(3)$ & $0.8407(3)$ & $0.1517(2)$ & $0.0196(4)$ \\
H10A & 0.4584 & 0.9185 & 0.1879 & $0.023^{*}$ \\
H10B & 0.2863 & 0.9183 & 0.1211 & $0.023^{*}$ \\
C11 & $0.5308(3)$ & $0.7311(3)$ & $0.0411(2)$ & $0.0260(5)$ \\
H11A & 0.5687 & 0.8125 & -0.0247 & $0.039^{*}$ \\
H11B & 0.4686 & 0.6612 & 0.0024 & $0.039^{*}$ \\
H11C & 0.6392 & 0.6488 & 0.0740 & $0.039^{*}$ \\
N1 & $0.0899(2)$ & $0.7153(2)$ & $0.56278(17)$ & $0.0159(3)$ \\
H1 & $0.030(4)$ & $0.814(4)$ & $0.576(3)$ & $0.019^{*}$ \\
O1 & $0.3601(2)$ & $0.71212(19)$ & $0.24936(14)$ & $0.0179(3)$ \\
O2 & $0.1685(2)$ & $0.9465(2)$ & $0.36622(16)$ & $0.0232(3)$ \\
C11 & $0.14126(8)$ & $0.04416(8)$ & $0.85625(5)$ & $0.02534(12)$ \\
I1 & $0.48122(2)$ & $0.28666(2)$ & $0.33716(2)$ & $0.01543(6)$ \\
& & & & \\
\hline
\end{tabular}

Atomic displacement parameters $\left(\AA^{2}\right)$

\begin{tabular}{lllllll}
\hline & $U^{11}$ & $U^{22}$ & $U^{33}$ & $U^{12}$ & $U^{13}$ & $U^{23}$ \\
\hline C1 & $0.0128(9)$ & $0.0169(9)$ & $0.0192(10)$ & $-0.0027(7)$ & $-0.0041(7)$ & $-0.0020(8)$ \\
C2 & $0.0143(9)$ & $0.0213(10)$ & $0.0191(10)$ & $-0.0020(8)$ & $-0.0017(7)$ & $-0.0036(8)$ \\
C3 & $0.0156(9)$ & $0.0278(11)$ & $0.0163(10)$ & $-0.0053(8)$ & $-0.0015(7)$ & $0.0002(8)$ \\
C4 & $0.0171(9)$ & $0.0168(9)$ & $0.0207(10)$ & $-0.0044(8)$ & $-0.0049(8)$ & $0.0049(8)$ \\
C5 & $0.0139(9)$ & $0.0160(9)$ & $0.0207(10)$ & $-0.0013(8)$ & $-0.0038(7)$ & $0.0001(8)$ \\
C6 & $0.0135(9)$ & $0.0170(9)$ & $0.0168(9)$ & $-0.0027(7)$ & $-0.0038(7)$ & $-0.0015(7)$ \\
C7 & $0.0126(8)$ & $0.0125(8)$ & $0.0171(9)$ & $-0.0024(7)$ & $-0.0021(7)$ & $-0.0009(7)$ \\
C8 & $0.0129(8)$ & $0.0163(9)$ & $0.0169(9)$ & $-0.0031(7)$ & $-0.0033(7)$ & $-0.0016(7)$ \\
C9 & $0.0144(9)$ & $0.0143(9)$ & $0.0195(10)$ & $-0.0038(7)$ & $-0.0025(7)$ & $-0.0004(7)$ \\
C10 & $0.0222(10)$ & $0.0162(9)$ & $0.0197(10)$ & $-0.0068(8)$ & $-0.0013(8)$ & $0.0043(8)$
\end{tabular}




\begin{tabular}{lllllll} 
C11 & $0.0347(12)$ & $0.0227(11)$ & $0.0205(11)$ & $-0.0121(10)$ & $0.0024(9)$ & $-0.0009(8)$ \\
N1 & $0.0139(8)$ & $0.0129(8)$ & $0.0186(8)$ & $-0.0010(6)$ & $-0.0016(6)$ & $-0.0018(6)$ \\
O1 & $0.0206(7)$ & $0.0125(6)$ & $0.0188(7)$ & $-0.0044(6)$ & $-0.0001(6)$ & $0.0020(5)$ \\
O2 & $0.0240(8)$ & $0.0135(7)$ & $0.0268(8)$ & $-0.0018(6)$ & $0.0023(6)$ & $-0.0001(6)$ \\
C11 & $0.0254(3)$ & $0.0228(3)$ & $0.0242(3)$ & $-0.0060(2)$ & $-0.0008(2)$ & $0.0092(2)$ \\
I1 & $0.01470(9)$ & $0.01248(9)$ & $0.01706(9)$ & $-0.00172(6)$ & $-0.00130(6)$ & $-0.00153(6)$ \\
\hline
\end{tabular}

Geometric parameters $\left(\AA,{ }^{\circ}\right)$

\begin{tabular}{|c|c|c|c|}
\hline $\mathrm{C} 1-\mathrm{N} 1$ & $1.361(3)$ & C7-I1 & $2.0660(19)$ \\
\hline $\mathrm{C} 1-\mathrm{C} 2$ & $1.405(3)$ & $\mathrm{C} 8-\mathrm{N} 1$ & $1.380(3)$ \\
\hline $\mathrm{C} 1-\mathrm{C} 6$ & $1.417(3)$ & $\mathrm{C} 8-\mathrm{C} 9$ & $1.463(3)$ \\
\hline $\mathrm{C} 2-\mathrm{C} 3$ & $1.384(3)$ & $\mathrm{C} 9-\mathrm{O} 2$ & $1.216(3)$ \\
\hline $\mathrm{C} 2-\mathrm{H} 2$ & 0.9500 & $\mathrm{C} 9-\mathrm{O} 1$ & $1.330(2)$ \\
\hline $\mathrm{C} 3-\mathrm{C} 4$ & $1.409(3)$ & $\mathrm{C} 10-\mathrm{O} 1$ & $1.453(2)$ \\
\hline $\mathrm{C} 3-\mathrm{H} 3$ & 0.9500 & $\mathrm{C} 10-\mathrm{C} 11$ & $1.515(3)$ \\
\hline $\mathrm{C} 4-\mathrm{C} 5$ & $1.376(3)$ & $\mathrm{C} 10-\mathrm{H} 10 \mathrm{~A}$ & 0.9900 \\
\hline $\mathrm{C} 4-\mathrm{Cl1}$ & $1.752(2)$ & $\mathrm{C} 10-\mathrm{H} 10 \mathrm{~B}$ & 0.9900 \\
\hline $\mathrm{C} 5-\mathrm{C} 6$ & $1.406(3)$ & $\mathrm{C} 11-\mathrm{H} 11 \mathrm{~A}$ & 0.9800 \\
\hline $\mathrm{C} 5-\mathrm{H} 5$ & 0.9500 & $\mathrm{C} 11-\mathrm{H} 11 \mathrm{~B}$ & 0.9800 \\
\hline $\mathrm{C} 6-\mathrm{C} 7$ & $1.421(3)$ & $\mathrm{C} 11-\mathrm{H} 11 \mathrm{C}$ & 0.9800 \\
\hline $\mathrm{C} 7-\mathrm{C} 8$ & $1.383(3)$ & $\mathrm{N} 1-\mathrm{H} 1$ & $0.77(3)$ \\
\hline $\mathrm{N} 1-\mathrm{C} 1-\mathrm{C} 2$ & $129.80(19)$ & $\mathrm{N} 1-\mathrm{C} 8-\mathrm{C} 9$ & $117.43(17)$ \\
\hline $\mathrm{N} 1-\mathrm{C} 1-\mathrm{C} 6$ & $108.08(18)$ & $\mathrm{C} 7-\mathrm{C} 8-\mathrm{C} 9$ & $133.80(19)$ \\
\hline $\mathrm{C} 2-\mathrm{C} 1-\mathrm{C} 6$ & $122.11(19)$ & $\mathrm{O} 2-\mathrm{C} 9-\mathrm{O} 1$ & $123.5(2)$ \\
\hline $\mathrm{C} 3-\mathrm{C} 2-\mathrm{C} 1$ & $117.05(19)$ & $\mathrm{O} 2-\mathrm{C} 9-\mathrm{C} 8$ & $122.96(19)$ \\
\hline $\mathrm{C} 3-\mathrm{C} 2-\mathrm{H} 2$ & 121.5 & $\mathrm{O} 1-\mathrm{C} 9-\mathrm{C} 8$ & $113.50(17)$ \\
\hline $\mathrm{C} 1-\mathrm{C} 2-\mathrm{H} 2$ & 121.5 & $\mathrm{O} 1-\mathrm{C} 10-\mathrm{C} 11$ & $106.61(17)$ \\
\hline $\mathrm{C} 2-\mathrm{C} 3-\mathrm{C} 4$ & $120.58(19)$ & $\mathrm{O} 1-\mathrm{C} 10-\mathrm{H} 10 \mathrm{~A}$ & 110.4 \\
\hline $\mathrm{C} 2-\mathrm{C} 3-\mathrm{H} 3$ & 119.7 & $\mathrm{C} 11-\mathrm{C} 10-\mathrm{H} 10 \mathrm{~A}$ & 110.4 \\
\hline $\mathrm{C} 4-\mathrm{C} 3-\mathrm{H} 3$ & 119.7 & $\mathrm{O} 1-\mathrm{C} 10-\mathrm{H} 10 \mathrm{~B}$ & 110.4 \\
\hline $\mathrm{C} 5-\mathrm{C} 4-\mathrm{C} 3$ & $123.23(19)$ & $\mathrm{C} 11-\mathrm{C} 10-\mathrm{H} 10 \mathrm{~B}$ & 110.4 \\
\hline $\mathrm{C} 5-\mathrm{C} 4-\mathrm{Cl} 1$ & $119.08(16)$ & $\mathrm{H} 10 \mathrm{~A}-\mathrm{C} 10-\mathrm{H} 10 \mathrm{~B}$ & 108.6 \\
\hline $\mathrm{C} 3-\mathrm{C} 4-\mathrm{Cl1}$ & $117.69(16)$ & $\mathrm{C} 10-\mathrm{C} 11-\mathrm{H} 11 \mathrm{~A}$ & 109.5 \\
\hline $\mathrm{C} 4-\mathrm{C} 5-\mathrm{C} 6$ & $117.06(19)$ & $\mathrm{C} 10-\mathrm{C} 11-\mathrm{H} 11 \mathrm{~B}$ & 109.5 \\
\hline $\mathrm{C} 4-\mathrm{C} 5-\mathrm{H} 5$ & 121.5 & $\mathrm{H} 11 \mathrm{~A}-\mathrm{C} 11-\mathrm{H} 11 \mathrm{~B}$ & 109.5 \\
\hline $\mathrm{C} 6-\mathrm{C} 5-\mathrm{H} 5$ & 121.5 & $\mathrm{C} 10-\mathrm{C} 11-\mathrm{H} 11 \mathrm{C}$ & 109.5 \\
\hline $\mathrm{C} 5-\mathrm{C} 6-\mathrm{C} 1$ & $119.95(19)$ & $\mathrm{H} 11 \mathrm{~A}-\mathrm{C} 11-\mathrm{H} 11 \mathrm{C}$ & 109.5 \\
\hline $\mathrm{C} 5-\mathrm{C} 6-\mathrm{C} 7$ & $133.51(19)$ & $\mathrm{H} 11 \mathrm{~B}-\mathrm{C} 11-\mathrm{H} 11 \mathrm{C}$ & 109.5 \\
\hline $\mathrm{C} 1-\mathrm{C} 6-\mathrm{C} 7$ & $106.53(18)$ & $\mathrm{C} 1-\mathrm{N} 1-\mathrm{C} 8$ & $109.37(17)$ \\
\hline $\mathrm{C} 8-\mathrm{C} 7-\mathrm{C} 6$ & $107.32(17)$ & $\mathrm{C} 1-\mathrm{N} 1-\mathrm{H} 1$ & $124(2)$ \\
\hline $\mathrm{C} 8-\mathrm{C} 7-\mathrm{I} 1$ & $129.30(15)$ & $\mathrm{C} 8-\mathrm{N} 1-\mathrm{H} 1$ & $127(2)$ \\
\hline C6- C7-I1 & $123.36(14)$ & $\mathrm{C} 9-\mathrm{O} 1-\mathrm{C} 10$ & $115.06(16)$ \\
\hline $\mathrm{N} 1-\mathrm{C} 8-\mathrm{C} 7$ & $108.70(18)$ & & \\
\hline $\mathrm{N} 1-\mathrm{C} 1-\mathrm{C} 2-\mathrm{C} 3$ & $179.0(2)$ & $\mathrm{C} 1-\mathrm{C} 6-\mathrm{C} 7-\mathrm{I} 1$ & $178.14(13)$ \\
\hline $\mathrm{C} 6-\mathrm{C} 1-\mathrm{C} 2-\mathrm{C} 3$ & $-1.6(3)$ & $\mathrm{C} 6-\mathrm{C} 7-\mathrm{C} 8-\mathrm{N} 1$ & $0.2(2)$ \\
\hline
\end{tabular}




\begin{tabular}{llll}
$\mathrm{C} 1-\mathrm{C} 2-\mathrm{C} 3-\mathrm{C} 4$ & $0.5(3)$ & $\mathrm{I} 1-\mathrm{C} 7-\mathrm{C} 8-\mathrm{N} 1$ & $-178.26(13)$ \\
$\mathrm{C} 2-\mathrm{C} 3-\mathrm{C} 4-\mathrm{C} 5$ & $0.4(3)$ & $\mathrm{C} 6-\mathrm{C} 7-\mathrm{C} 8-\mathrm{C} 9$ & $176.9(2)$ \\
$\mathrm{C} 2-\mathrm{C} 3-\mathrm{C} 4-\mathrm{C} 11$ & $179.75(16)$ & $\mathrm{I} 1-\mathrm{C} 7-\mathrm{C} 8-\mathrm{C} 9$ & $-1.5(3)$ \\
$\mathrm{C} 3-\mathrm{C} 4-\mathrm{C} 5-\mathrm{C} 6$ & $-0.2(3)$ & $\mathrm{N} 1-\mathrm{C} 8-\mathrm{C} 9-\mathrm{O} 2$ & $1.2(3)$ \\
$\mathrm{C} 11-\mathrm{C} 4-\mathrm{C} 5-\mathrm{C} 6$ & $-179.59(15)$ & $\mathrm{C} 7-\mathrm{C} 8-\mathrm{C} 9-\mathrm{O} 2$ & $-175.3(2)$ \\
$\mathrm{C} 4-\mathrm{C} 5-\mathrm{C} 6-\mathrm{C} 1$ & $-0.8(3)$ & $\mathrm{N} 1-\mathrm{C} 8-\mathrm{C} 9-\mathrm{O} 1$ & $-179.20(16)$ \\
$\mathrm{C} 4-\mathrm{C} 5-\mathrm{C} 6-\mathrm{C} 7$ & $-179.7(2)$ & $\mathrm{C} 7-\mathrm{C} 8-\mathrm{C} 9-\mathrm{O} 1$ & $4.3(3)$ \\
$\mathrm{N} 1-\mathrm{C} 1-\mathrm{C} 6-\mathrm{C} 5$ & $-178.72(18)$ & $\mathrm{C} 2-\mathrm{C} 1-\mathrm{N} 1-\mathrm{C} 8$ & $179.1(2)$ \\
$\mathrm{C} 2-\mathrm{C} 1-\mathrm{C} 6-\mathrm{C} 5$ & $1.7(3)$ & $\mathrm{C} 6-\mathrm{C} 1-\mathrm{N} 1-\mathrm{C} 8$ & $-0.4(2)$ \\
$\mathrm{N} 1-\mathrm{C} 1-\mathrm{C} 6-\mathrm{C} 7$ & $0.5(2)$ & $\mathrm{C} 7-\mathrm{C} 8-\mathrm{N} 1-\mathrm{C} 1$ & $0.1(2)$ \\
$\mathrm{C} 2-\mathrm{C} 1-\mathrm{C} 6-\mathrm{C} 7$ & $-179.05(18)$ & $\mathrm{C} 9-\mathrm{C} 8-\mathrm{N} 1-\mathrm{C} 1$ & $-177.20(17)$ \\
$\mathrm{C} 5-\mathrm{C} 6-\mathrm{C} 7-\mathrm{C} 8$ & $178.7(2)$ & $\mathrm{O} 2-\mathrm{C} 9-\mathrm{O} 1-\mathrm{C} 10$ & $2.2(3)$ \\
$\mathrm{C} 1-\mathrm{C} 6-\mathrm{C} 7-\mathrm{C} 8$ & $-0.4(2)$ & $\mathrm{C} 8-\mathrm{C} 9-\mathrm{O} 1-\mathrm{C} 10$ & $-177.42(16)$ \\
$\mathrm{C} 5-\mathrm{C} 6-\mathrm{C} 7-\mathrm{I} 1$ & $-2.8(3)$ & $\mathrm{C} 11-\mathrm{C} 10-\mathrm{O} 1-\mathrm{C} 9$ & $-178.33(17)$ \\
\hline
\end{tabular}

Hydrogen-bond geometry $\left(\AA,{ }^{\circ}\right)$

\begin{tabular}{lllll}
\hline$D-\mathrm{H} \cdots A$ & $D-\mathrm{H}$ & $\mathrm{H} \cdots A$ & $D \cdots A$ & $D-\mathrm{H} \cdots A$ \\
\hline $\mathrm{N} 1-\mathrm{H} 1 \cdots \mathrm{O} 2^{\mathrm{i}}$ & $0.77(3)$ & $2.06(3)$ & $2.821(2)$ & $168(3)$ \\
\hline
\end{tabular}

Symmetry code: (i) $-x,-y+2,-z+1$. 